\title{
LXXIII. On light
}

\section{Andrew Ure M.D.}

To cite this article: Andrew Ure M.D. (1821) LXXIII. On light, Philosophical Magazine Series 1, 57:278, 409-418, DOI: 10.1080/14786442108652535

To link to this article: http://dx.doi.org/10.1080/14786442108652535

$$
\text { 曲 Published online: } 27 \text { Jul } 2009 .
$$

Submit your article to this journal

LII Article views: 4

Q View related articles ¿ 
LXXIII. On Light. By ANDnEw URE,M.D. Professor of the Andersonian Institution, Glasgow*.

Lrght. The agent of vision.-Some philosophers regard light as consisting of particles of inconceivable minuteness, emitted in succession by luminous bodies, which move in straight lines, at the rate of 200,000 m:les per second.

Others conceive that it consists in certain undulations communicated by luminous bodies, to an ethereal fluid which fills all space. This fluid is composed of the most subtile matter, is highly elastic, and the undulations are propagated through it with great velocity, in spherical superficies proceeding from a centre. This view derives great plausibility from its happy application by Huygens, to explain a very difficult class of optical phænomena, the double refraction of calcareous spar and other bodies.

The common refraction is explained by Huygens on the supposition, that the undulations in the luminous fluid are propagated in the form of spherical waves. The double refraction is explained on the supposition, that the undulations of light, in passing through the calcareous spar, assume a spheroidal form; and this hypothesis, though it does not apply with the same simplicity as the former, yet admits of such precision, that a proportion of the axes of the spheroids may be assigned, which will account for the precise quantity of the extraordinary refraction, and for all the phænomena dependent on it, which Huygens had studied with great care; and had reduced to the smallest number of general facts.

"That these spheroidal undulations actually exist," says the celebrated Playfair, "he would after all be a bold theorist who should affirm; but that the supposition of their existence is an accurate expression of the phænomena of double refraction, cannot be doubted. When one enunciates the hypothesis of the spheroidal undulations, he in fact expresses in a single sentence all the phænomena of double refraction. The hypothesis is therefore the means of representing these phænomena, and the laws which they obey, to the imagination or the understanding; and there is perhaps no theory in optics, and but very few in natural philosophy, of which more can be said. Theory therefore, in this instance, is merely to be regarded as the expression of a general law, and in that light I think it is considered by Laplace."

Dr. Young has selected from Sir Isaac Newton's various writings, many passages favourable to the admission of the undulatory theory of light, or of a luminiferous ether pervading the uni-

* From Ure's Dictionary of Chemistry.

Vol.57. No. 278. June 1821. $3 \mathrm{~F}$

verse, 
verse, rare and elastic in a high degree. "Is not the heat (of the warm room) conveyed through the vacuum by the vibrations of a much subtiler medium than air? And is not this medium the same with that medium by which light is reflected and refracted, and by whose vibrations light communicates heat to bodies, and is put into fits of easy reflection and easy transmission? And do not the vibrations of this medium in hot bodies contribute to the intenseness and duration of their heat ? And do not hot bodies communicate their heat to contiguous cold ones, by the vibrations of this medium, propagated from them into the cold ones? And is not this medium exceedingly more rare and subtile than the air, and exceedingly more elastic and active? And doth it not readily pervade all bodies? And is it not by its elastic force expanded through all the heavens?""If any one would ask how a medium can be so rare, let him tell me how an electric body can by friction emit an exhalation so rare and subtile, and yet so potent? and how the effluvia of a magnet can pass through a plate of glass without resistance, and yet turn a magnetic needle beyond the glass?"-Optics, $Q u$. 18,22 . "Were I to assume an hypothesis, it should be this, if propounded more generally, so as not to determine what light is, further than that it is something or other capable of exciting vibrations in the ether; for thus it will become so general, and comprehensive of other hypotheses, as to leave little room for new ones to be invented." - Birch, iii. 249.

Dr. Young shows, that many phænomena inexplicable on the notion of radiating corpuscles, are easily reconciled to the theory of undulation. "On the whole," says this profound philosopher, " it appears that the few optical phænomena, which admit of explanation by the corpuscular system, are equally consistent with this theory; that many others which have been long known, but never understood, become by these means perfectly intelligible; and that several new facts are found to be thus, only, reducible to a perfect analogy with other facts, and to the simple principles of the undulatory system."-Nat. Phil. vol. ii. p. 631.

I think, however, that the new discoveries on polarized light may be more easily referred to the corpuscular than undulatory hypothesis.

The physical affections of light are foreign to this work. Its chemical relations are alone to be considered. These may be conveniently referred to four heads :

l. Of the mean refractive and dispersive powers of different bodies.

2. Of the action of the different prismatic colours on chemical matter.

3 . Of the polarization of light. 
4. Of the absorption and disengagement of light or phospho. rescence.

1. Newton first discovered that certain bodies exercise on light a peculiar attractive force. When a ray passes obliquely from air into any transparent liquid or solid surface, it undergoes at estrance an angular flexure, which is called refraction. The variation of this departure from the rectilineal path for any particular substance, depends on the obliquity of the ray to the refracting surface; so that the sine of the angle of refraction, is to that of the angle of incidence, in a constant ratio. Now Newton found, that unctuous or inflammable bodies occasioned a greater deviation in the luminous rays than their attractive mass or density gave reason to expect. Hence he conjectured, that both diamond and water contained combustible matter, - a sagacious anticipation of future chemical discovery.

Dr. Wollaston invented a very ingenious apparatus, in which, by means of a rectangular prism of flint glass, the index of refraction of each substance is read off at once by a vernier, the three sides of a moveable triangle performing the operations of reduction, in a very compendious manner.-Phil. Trans. 1802, or Nicholson's Journal, 8vo. vol. iv. p. 89.

But transparent media occasion not merely a certain flexure of the white sunbeam, called the mean refraction, they likewise decompose it into its constituent colours. This effect is called dispersion. Now the mean refractive and dispersive powers of bodies are not proportional to each other. In some refracting media, the mean angle of refraction is larger, whilst the angle of dispersion is smaller; and in other refracting media, the mean angle of refraction is smaller, whilst the angle of dispersion is larger. In short, the knowledge of the mean refractive power of a given substance, will not enable us to determine its dispersive power, and vice versâ.

From the refractive power of bodies we may in many cases infer their chemical constitution. For discovering the purity of essential oils, an examination with Dr. Wollaston's instrument may be of considerable utility, on account of the smallness of the quantity requisite for trial. "In oil of cloves, for instance, I have met with a wide difference. The refractive power of genuine oil of cloves is as high as 1.535 ; but $I$ have also purchased oil by this name which did not exceed $1 \cdot 498$, and which had probably been adulterated by some less refractive oil." This tine idea, suggested by Dr. Wollaston, has been happily prosecuted by M. Biot, with regard to gaseous compounds. I shall first give general tables of the refractive and dispersive powets of different bodies, and then make some remarks on their chemical applications: 
Index of Refraction.

Index of Refraction.

\begin{tabular}{ll|l} 
A vacuum, & 1.00000 & English plate-glass, W. 1.504
\end{tabular} $\left.\begin{array}{c}\text { Atmospheric air, } \\ \text { (mean,) }\end{array}\right\} \quad 1 \cdot 00033 \begin{aligned} & \text { Oil of amber, } \\ & \text { Balsam of capivi, }\end{aligned}$ W. W. 1.505

Ice,

Ice, $\int_{\text {W. }} 1 \cdot 31000$

Brewster, $1 \cdot 30700$

Gum-arabic, W. 1.514

Water, 1.336

Vitreous Cryo-

humour, $\int$ lite. B. $1 \cdot 344$

Ether,

Wol. $1 \cdot 353$

Albumen,

W. $1 \cdot 360$

Alcohol,

W. $1 \cdot 370$

Saturated solution Cavallo, $\quad \mathbf{1} 375$ of salt, $\left.\begin{array}{l}\text { Solution of sal am- } \\ \text { moniac, }\end{array}\right\} \quad 1.382$

Nitric acid, sp.gr.
$1 \cdot 48$, W. $1 \cdot 410$ 1.48 ,

Fluor spar,

W. $1 \cdot 433$

Sulphuric spar, W. 1.435

Spermaceti melted, W. $1 \cdot 446$

Crystalline lens? of an ox, $\}$

Alum,

W. $1 \cdot 447$

Tallow melted,

W. $1 \cdot 457$

Borax,

W. $1 \cdot 460$

Oil of lavender,

C. $1 \cdot 467$

W. $1 \cdot 467$

C. $(1 \cdot 469)$

Oil of peppermint, W. $1 \cdot 468$

Oil of olives, W. $1 \cdot 469$

Oil of almonds, W. $1 \cdot 470$ rectified,

Do. common, W. 1.476

Essence of lemon, W. $1 \cdot 476$

Butter, cold,

Linseed oil,

W. $1 \cdot 48($ )

Camphor,

W. $1 \cdot 485$

Iceland spar,

W. $1 \cdot 487$ weakest refr. $\}$ W. 1.488

Do. strongest do.

Tallow, cold,

W. $(1 \cdot 657)$

Sulphate of potash, W. $1 \cdot 495$

Oil of nutmeg, W. $1 \cdot 497$

French plate-glass, W. 1·500

Dutch plate-glass, W. 1.517

Caoutchouc, W. 1.524

Nitre,

C. $1 \cdot 524$

Selenite,

W. $1 \cdot 525$

Crown glass, com. W. 1.525

Canada balsam, W. $1 \cdot 528$

Centre of the cry-7 stalline of fish, and dry crystalline of an ox,

Pitch,

W. $1 \cdot 530$

Radcliffe crown-?

glass,

Anime,

W.

Copal,

W. 1.533

Oil of cloves,

W. 1.535

White wax, cold,

Elemi,

Mastic,

Arseniate of potash, W.

Sugar after fusion,

Spermaceti, cold,

Red sealing-wax,

Oil of sassafras,

W. 1.536

Bees-wax,

W. $1 \cdot 542$

Boxwood,

W.

Colophony, W. $1 \cdot 543$

Old plate-glass, W. 1.545

Rock cry'stal, (double,

Amber,

W. $1 \cdot 547$

W. $1 \cdot 547$

Opium,

C. $(1 \cdot 556)$

Mica,

W.

Phosphorus,

W.

Horn,

Flint-glass,

Benzoin,

W. $1 \cdot 579$

W.

Guaiacum,

W. $\left\{\begin{array}{l}1.583 \\ 1.586\end{array}\right.$

W.

Balsam of Tolu, W. $1 \cdot 600$ 
Index of Refraction.

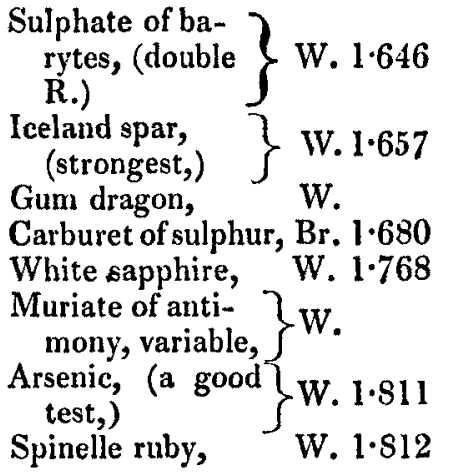

Index of Refraction.

Jargon, W. $1 \cdot 950$

Glass of antimony, W. $1 \cdot 980$

Native sulphur, W. $20 \cdot 40$

Do. Brewster, 2.115

Plumbago, W.

Phosphorus, Brewster, 2·224

Diamond, $\left\{\begin{array}{l}\text { Newton, } \\ \text { by Dr. W. } 2 \cdot 440\end{array}\right.$

Do. Rochon, 2.755

Realgar, Brewster, 2.510

Chromate of lead, ?

(least refr.)

do. $24 \cdot 79$

Do. (greatest refr.) do. $2 \cdot 926$

Table II. - Refracting Powers of Gases for the Temperature of $32^{\circ} \mathrm{F}$. and Pressure 30, by MM. Brot and ARAgo.

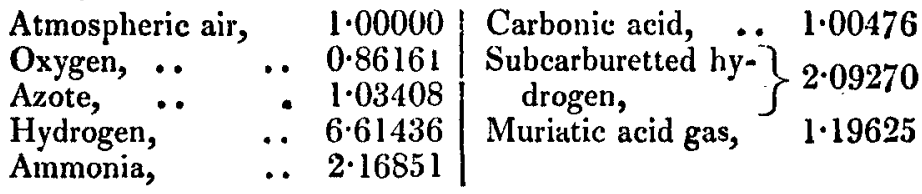

TABLE III.-Dispersive Powers.

Cryolite, Brewster, 0.022 Sulphur, Brewster, 0.130

Fluor spar, do. 0.022 Oil of cassia, .. do. 0.139

Water, .. do. 0.035 Realgar, .. do. 0.255

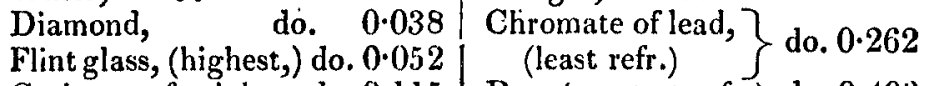

Carburet of sulphur, do. $0 \cdot 115$ Do. (greatest refr.) do. 0.400

Phosphorus, do. $0 \cdot 128$

Carburet of sulphur exceeds all fluid bodies in refractive power, surpassing even flint-glass, topaz, and tourmaline; and in dispersive power it exceeds every fluid substance, except oil of cassia, holding an intermediate place between phosphorus and balsam of Tolu.

Dr. Brewster has further shown, that all doubly refracting crystals have two dispersive powers.

From Table II. it appears, that the refractive power of hydrogen gas greatly surpasses not only that of the other gases, but of all known bodies. 'This principle exists in great abundance, in resins, oils, and gums, where it is united to carbon and oxygen; and we must probahly ascribe to it, the eminent refractive power of these combustibles, so justly observed by Newton. This effect of hydrogen is finely displayed in ammonia, whose refractive power 
is more than double that of air ; and much superior to that of water.

But since every substance ought to introduce into its combinations, its peculiar character, and preserve in them, to a certain degree, the force with which it acts on light, let us endeavour to calculate, in this point of view, the refractive influence of the constituents of a compound. From our knowledge of the extreme tenuity of light, it is probable, that the influence of a moderate chemical condensation, ought to affect its operations very slightly; for, whether it be an ether or a corpuscular emanation, the excessive minuteness of its particles, compared to the distances between the molecules of bodies, ought to render the change of distance among the latter, unimportant. Consequently, the refracting powers of bodies ought to differ very little from those of their elements, unless a very great degree of condensation has taken place.

Hence, if we multiply the proportions of azote and oxygen respectively, by their refractive powers, we shall obtain products, whose sums will coincide with the refractive power of the atmosphere. Thus, 100 parts by weight of the atmosphere, consist of azote $77 \cdot 77+$ oxygen $22 \cdot 22$. If we multiply each of these numbers by the number representing the refractive power of the body, and making a small correction for the carbonic acid present, we shall have for the sum of the products 1.0000.

Ammonia, however, furnishes a more interesting example of the application of these principles.

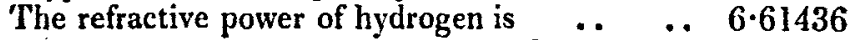

$$
\begin{array}{lll}
\text { of azote } & 1 \cdot 03408 \\
\text { of ammonia } & 2 \cdot 16851
\end{array}
$$

Let $x$ be the weight of the constituent, whose refractive power is

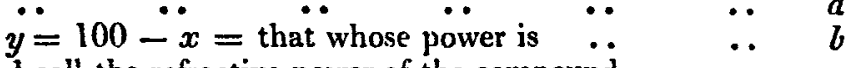

$$
\begin{aligned}
& \text { and call the refractive power of the compound .. } c \\
& \text { Then } x=\frac{c-b}{a-b} \text {. In the present case, } \\
& x=\frac{2 \cdot 16851-1 \cdot 03408}{6 \cdot 61436-1 \cdot 03408}=0.203 \text { and } 100-x=0.797=
\end{aligned}
$$

the azote in 100 parts of ammonia; which may be regarded as an approximation. The true proportions given by the equivalent ratios are, 0.823 azote +0.177 hydrogen. If the refractive power of ammonia were 2.0218 , then the chemical and optical analysis would coincide.

If we calculate on the above data, what ought to be the refractive power of water, as a compound of 8 parts of oxygen + 1 hydrogen, we shall obtain the number $1 \cdot 50065$, which being multiplied 
multiplied by 0.45302 , the absolute refractive power of air, when we take the density of water for unity, we shall have a product $=0.67984$. Now, according to Newton's estimate, which M. Biòt has found to be exact, the refractive power of water is 0.7845 . Hence we see that the compound has acquired an increased refractive force by condensation, above the mean of its constituents, in the ratio of 100 to $86 \frac{2}{3}$.

Rays of light, in traversing the greater number of crystallized bodies, are commonly split into two pencils ; one of which, called the ordinary ray, follows the common laws of refraction, agreeably to the preceding tables, whilst the other, called the extraordinary ray, obeys very different laws. This phæuomenon is produced in all transparent crystals, whose primitive form is neither a cube nor a regular octohedron. The division of the beam is greater or less, according to the nature of the crystal, and the direction in which it is cut. But of all known substances, that which produces this phænomenon in the most energetic manner, is the rhomboidal carbonate uf lime commonly called island spar.

2. Of the action of the different coloured rays. If the white sunbeam, admitted through a small hole of a window-shutter into a darkened room, be made to pass through a triangular prism of glass, it will be divided into a number of splendid colours which may be thrown upon a sheet of paper. Newton ascertained, that if this coloured image, or spectrum as it is called, be divided into 360 parts, the red will occupy 45 , the orange 27 , the yellow 48 , the green 60 , the blue 60 , the indigo 40 , and the violet 80 . The red rays being least bent by the prism, from the direction of the white beam, are said to be least refracted, or the least refrangible ; while the violet rays being always at the other extremity of the spectrum, are called the most refrangible. According to Dr. Wollaston, when the beam of light is only 1-20th of an inch broad, and received by the eye at the distance of 10 feet, through a clear prism of flint-glass, only four colours appear; red, yellowish-green, blue, and violet.

If the differently coloured rays of light thus separated by the prism, be concentred on one spot by a lens, they will reproduce colourless light. Newton ascribes the different colours of bodies, to their power of absorbing all the primitive colours, except the peculiar one which they reflect, and of which colour they therefore appear to our eye.

According to Sir William Herschel, the different coloured rays possess very different powers of illumination. The lightest green, or deepest yellow, which are near the centre, throw more light on a printed page than any of the rays towards either side of the spectrum. Sir H. Davy remarks, that as there are more greet rays in a given part of the spectrum than blue rays, the difference 
of illuminating power may depend on this circumstance. The rays separated by one prism, are not capable of being further divided by being passed through another; and in their relations to double refraction and reflection, they appear to agree with direct fight. An ohject illuminated by any of the rays in the spectrum, is seen double through island crystal, in the same manner as if it had been visible by white light.

Under Caloric, we have stated the power of heating which the different coloured rays of the spectrum apparently possess. Sir H. Englefield, and M. Berard, confirmed the results of Sir $W$. Herschel, with regard to the progressive increase of calorific infuence from the violet to the red extremity of the spectrum; and they also found with him, that a calorific influence extended beyond the limit of the red light, into the unilluminated space. M. Berard, however, observed, that the maximum of effect was in the light, and not beyond it. This ingenious philosopher made a pencil of the sunbeam pass across a prism of island spar. The division of the rays formed two spectra, which presented the same properties with the single spectrum. Both possessed the calorific virtue in the same manner and degree. M. Berard polarized a beam of light by refection from a mirror; and he found that in all the positions in which light ceased to be reflected, heat also ceased to appear. The thermometer in the focus of the apparatus was no longer affected. Thus, we see that the obscure heatmaking principle accompanies the luminous particles, and obeys the same laws of action.

If the white luna cornea, the muriate of silver moistened, be exposed to the different rays in the prismatic spectrum, it will be found, that no effect is produced upon it, in the least refrangible rays, which occasion heat without light ; that only a slight discoloration will be occasioned by the red rays; that the blackening power will be greater in the violet than in any other ray; and that beyond the violet, in a space perfectly obscure to our eyes, the darkening effect will be manifest on the muriate.

This fine observation, due to M. Ritter and Dr. Wollaston, proves, that there are rays more refrangible than the rays producing light and heat. As it appears, from the observations of M. Berthollet, that muriatic acid gas is formed when horn-silver is blackened by light, the above rays may be called hydrogenating. Sir H. Davy found, that a mixture of chlorine and hydrogen acted more rapidly upon each other, combining without explosion, when exposed to the red rays, than when placed in the violet rays ; but that solution of chlorine in water became solution of nuriatic acid most rapidly, when placed in the most refrangible rays in the spectrum. He also observed, that the puce-coloured oxide of lead, when moistened, gradually gained a tint of red in the 
least refrangible rays, and at last became black, but was not affected in the most refrangible rays. The same change was produced by exposing it to a current of hydrogen gas. The oxide of mercury from calomel and water of potash, when exposed to the spectrum, was not changed in the most refrangible rays, but became red in the least refrangible; which must have been owing to the absorption of oxygen. The violet rays produced upon moistened red oxide of mercury, the same effect as hydrogen gas.

Dr. Wollaston found, that guaiac, exposed to the violet rays, passed rapidly from yellow to green; and MM. Gay Lussac and Thenard applied to the same influence a gaseous mixture of hydrogen and chlorine; when explosion immediately took place. By placing small bits of card, coated with moist horn-silver, or little phials of those nixed gases, in the different parts of the spectrum, M. Berard verified the former observations of the chemical power acquiring a maximum in the violet ray, and existing even beyond it; but he also found, that by leaving the tests a sufficient time in the indigo and blue rays, a perceptible effect was produced upon them. He concentrated by a lens all that portion of the spectrum which extends from the green to the extreme boundary of the violet; and by another lens he collected the other half of the spectrum, comprehending the red. The latter formed the focus of a white light, so brilliant, that the eye could not endure it; yet in two hours it produced no sensible change on muriate of silver. On the contrary, the focus of the other half of the spectrum, whose light and heat were far less intense, blackened the muriate in ten minutes. The investigations of Delaroche enable us, in some measure, to reduce these dissimilar effects of light to a common principle. See CaLoric.

In Mr. Brande's late Bakerian lecture on the composition and analysis of coal and oil gases, this ingenious chemist shows, that the light produced by these, or by olefiant gas, even when concentrated so as to produce a sensible degree of heat, occasioned no change on the colour of muriate of silver, nor on a mixture of chlorine and hydrogen; while the light emitted by electrized charcoal, speedily affects the muriate, catises these gases to unite rapidly, and sometimes with explosion. The conceritrated light of the moon, like that of the gases, produced no change. He concludes with stating, that he found the photometer of Mr. Leslie ineffectual. He employed one filled with the vapour of ether (renewable from a column of that fluid), which he found to be more delicate.

The general facts, says Sir H. Davy, of the refraction and effects of the solar beam, offer an analogy to the agencies of electricity. In the voltaic circuit, the maximum of heat seems to be at the positive pole, where the power of combining with oxygen Vol. 57. No. 278. June 1821 . $3 \mathrm{G}$ 
is given to bodies, and the agency of rendering bodies infiammable is exerted at the opposite surface; and similar chemical effects are produced by negative electricity, and by the most refrangible rays of the solar beam. In general, in nature, the effects of the solar rays are very compounded. Healthy vegetation depends upon the presence of the solar beams, or of light; and whilst the heat gives fluidity and mobility to the vegetable juices, ehemical effects likewise are occasioned, oxygen is separated from them, and inflammable compounds formed. Plants deprived of light become white, and contain an excess of saccharine and aqueous particles; and flowers owe the variety of their hues to the influence of the solar beams. Even animals require the presence of the rays of the sun, and their colours seem materially to depend upon the chemical infuence of these rays: a comparison between the polar and tropical animals, and between the parts of their bodies exposed, and those not exposed to light, shows the correctness of this opinion.

[To be continued.]

\section{Olservations on cerlain luminous Meteors called Falling} Stars. By Dr.T. Forster, M.B. F.L.S. ËC.

T Hartwell, E. Grinstead, June 2, 1821. Sir, - I observed this morning the Queries of Mr. J. Farey Sen., respecting Shooting Stars, as they are commonly called; and I beg leave through your useful Magazine, to communicate such information and references to this subject as at present occur to me. I have for many years been an attentive observer of these curious meteors, and have noticed their peculiarities, and the particular states of the atmosphere in which they occur, with a view to ascertain their nature, and their relation to electrical phænomena.

On the large neteors, such, for example, as the great meteor of August 18, 1783, many treatises have been written, to which I have referred in a small work on atmospheric phænomena*. I have there alluded to the probable connexion between these phænomena, and the aërolites called Meteoric Stones, and to the curious observations of the late M. J. A. De Luc on this subject.

How far the smaller meteors called Falling Stars may be referable to similar causes, I am at present unable to say; neither would I venture, in the present stage of the inquiry, to adopt any particular hypothesis to explain them. The first object in meteorology, as in all sciences, must be to obtain an accurate series of facts well arranged. With this view, I have for upwards of

* Researches about atmospheric Phænomena, by T. Forster, London, $1814,2 d$ edition, p. 114. 